\title{
Modeling GeV Observations of Gamma-ray Bursts
}

\author{
Rudy Gilmore* \\ SISSA - Astrophysics Sector - Via Bonomea 265 - 34136, Trieste - Italy \\ E-mail: rgilmoredsissa.it
}

\section{Aurelien Bouvier}

Santa Cruz Institute for Particle Physics, University of California, Santa Cruz, CA 95064, USA

\section{Nepomuk Otte}

Santa Cruz Institute for Particle Physics, University of California, Santa Cruz, CA 95064, USA

\section{Joel R. Primack}

Department of Physics, University of California, Santa Cruz, CA 95064, USA

\begin{abstract}
Fermi has shown GRBs to be a source of $>10 \mathrm{GeV}$ photons. We present an estimate of the detection rate of GRBs with the future Cherenkov Telescope Array (CTA). Our predictions are based on the observed properties of GRBs detected by Fermi, combined with the spectral properties and redshift determinations for the bursts population by instruments operating at lower energies. We develop two model for high energy prompt and early afterglow emission, and show how the probability of detection is affected by instrument effective area, response time, and energy threshold. While detection of VHE emission from GRBs has eluded ground-based instruments thus far, our results suggest that ground-based detection may be within reach of CTA, though detections would be infrequent even with prompt followup to all valid satellite triggers. We estimate a rate of one GRB every $2-3$ years based on the trigger rate from the Swift satellite, provided that no spectral softening or cutoff features below $100 \mathrm{GeV}$ exist in a significant number of GRBs. Such a detection would help constrain the emission mechanism of gamma-ray emission from GRBs. Photons at these energies from distant GRBs are affected by the UV-optical background light, and a ground-based detection could also provide a valuable probe of the Extragalactic Background Light (EBL) in place at high redshift.
\end{abstract}

25th Texas Symposium on Relativistic Astrophysics - TEXAS 2010

December 06-10, 2010

Heidelberg, Germany

\footnotetext{
${ }^{*}$ Speaker.
} 


\section{Introduction}

The observation of gamma-ray bursts (GRBs) with ground-based imaging atmospheric Cherenkov telescopes (IACTs) has been a tantalizing possibility in recent years. Powerful $>10$-meter telescope arrays such as H.E.S.S., MAGIC, and VERITAS have come online in the last decade, and satellite detectors such as Swift are capable of providing the necessary localization of events within seconds. Despite major campaigns to respond to satellite burst alerts at all three of these instruments (e.g., [1, 2, 3]), no conclusive detection of a GRB with an IACT has yet been made. However, observations of GRBs with the LAT instrument on the Fermi satellite have provided new insight into the emission of GRBs in the VHE band. Since its launch on June 11, 2008, Fermi LAT has detected GRBs at $>100 \mathrm{MeV}$ energies at a rate of about 10/yr. The highest energy detected photons have had rest frame energies exceeding $90 \mathrm{GeV}$.

In a recent paper [4] (GPP10), we addressed the possibility of detecting GeV-scale emission from GRBs with the Fermi LAT and MAGIC telescopes. Using simple assumptions about the GRB rate and spectral extrapolation based on the Swift GRB population, this work predicted the number of GRBs that could be detected per year, and the likely number of photons events per background for each case. GPP10 predicted the Fermi LAT to have a detection rate of 3-4 GRBs/yr above $10 \mathrm{GeV}$ per year, a rate that was matched well in the first year of telescope operations, but may have since proven to be an overestimate. The detection rate for MAGIC, ignoring instrumental background, was calculated to be 0.2-0.3 events per year, after somewhat optimistic assumptions were made about the telescope response time to satellite burst triggers. While the rate predicted for MAGIC was low, and may explain the lack of detections by the current generation of ground-based instruments, the predicted gamma-ray rates for a GRB detection near zenith were found to be up to thousands of counts in the lower energy range of the experiment within the prompt and early afterglow phases of the GRB, a large potential scientific payoff.

Here, we describe a new calculation with a more sophisticated treatment of the spectral extrapolation to high energies and the telescope detection capabilities. We have also implemented the $t^{-1.5}$ afterglow feature that has been seen in several GRBs by LAT [5], and may more realistically represent the signal visible at $\mathrm{GeV}$ energies. In the next section, we describe the ingredients and motivation of our model for high energy emission.

\section{Methods}

To a large extent, the challenge of modeling GRBs arises out of the large variance in properties seen between bursts, and the lack of a simple model describing the radiative mechanism. In GPP10, our extrapolation of GRB emission to high energies was based on the assumption that flux at VHE energies could be described as a fixed fraction of the flux at lower energies. In that work, a flux ratio of

$$
\frac{F(100 \mathrm{MeV}-5 \mathrm{GeV})}{F(20 \mathrm{keV}-2 \mathrm{MeV})}=0.1
$$

was assumed, reflecting the typical ratio inferred from coincident BATSE/EGRET observations. While photon statistics for EGRET-observed GRBs were severely limited, LAT observations have now given us an opportunity to reexamine our assumptions about these flux correlations. 

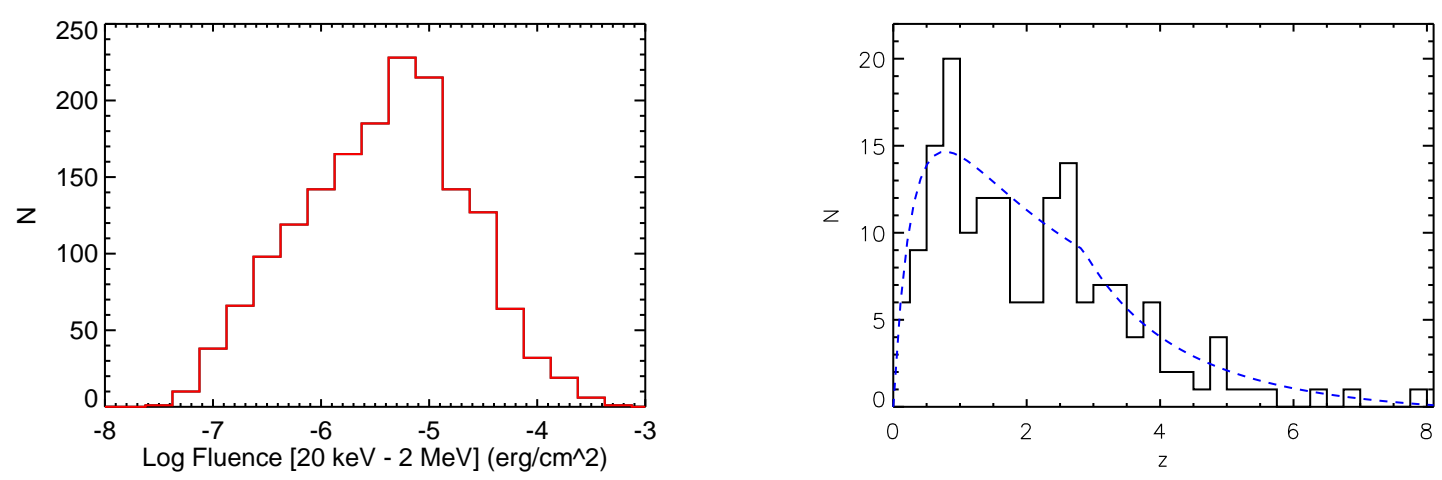

Figure 1: Left: Histogram of total fluence in the BATSE energy range for our sample of GRBs. Right: The redshifts determined for Swift GRBs. The dashed line shows the fit used in this work.

In this work, we have turned to the large sample of GRBs available from the BATSE catalog, providing the GRB fluence distribution and Band [6] spectral fits used in our analysis which we briefly describe in this section. The redshift distribution of GRBs is assumed to follow that of the Swift GRB population. Figure 1 shows input distributions for fluence and redshift used in our work. Attempting to build a sample of GRBs for analysis from populations of two different instruments will necessarily introduce biases. These biases will be studied in a upcoming publication and included amongst a comparison of different populations. Our calculation includes the impact of attenuation by UV-optical background photons on GRB emission using the fiducial model of [7].

\subsection{Spectral extrapolation}

Each of the 4 bright GRBs seen by LAT above $10 \mathrm{GeV}$ shows differing behavior. GRB 080916C, the first such detection by LAT (seen about 3 months after launch), was found to be well-described in all time bins by a continuation of the Band function determined at GBM energies [8]. Separate spectral components from the Band function were found to be required to match the GeV-scale emission of the 3 other brightest GRBs in the LAT catalog, long-duration GRBs 090902B and 090926, and short burst 090510. We have therefore considered two methods of extrapolating the lower energy emission to higher energies in this work.

As a minimal model, we consider the visibility predicted for GRBs at high energy without any significant deviation from the Band fit. In this model, labeled 'bandex' below, the high-energy spectrum is simply a continuation of the Band spectra determined at lower energy. The high energy normalization is therefore determined by the Band function peak energy, normalization, and the upper energy index $\beta$, which continues to $\mathrm{GeV}$ energies. We have enforced the requirement that $\beta$ not be harder than -2 , and the bursts in the sample with harder spectra are reset to this value.

In the fixed-parameter ("fixed") model, we make the assumption that the fluence between BATSE (20 keV to $2 \mathrm{MeV}$ ) energies and $\mathrm{GeV}$-scale (100 MeV to $10 \mathrm{GeV}$ ) energies can be described by a single ratio. We use here a flux ratio of 0.1 , which is well supported by observations of GBM-LAT observations of long-duration GRBs [11]. The spectral index at high energies is set to -2 , consistent with the mean value for EGRET GRBs of $-1.95[12,13]$, and near the center of the distribution for LAT-detected events [5]. Both of these values are quite similar to the prompt 


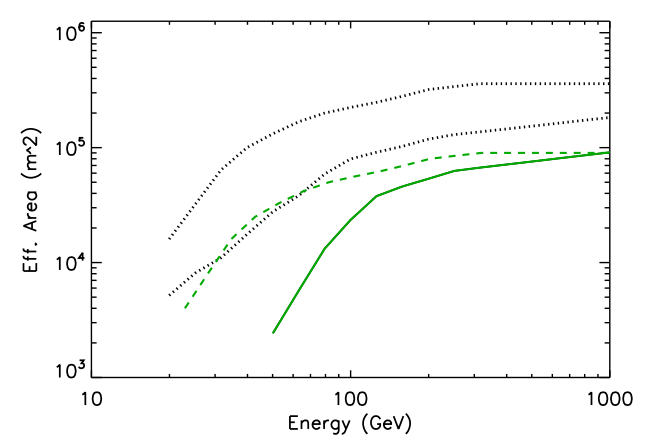

Figure 2: The effective area functions used in this work. Solid green is the MAGIC effective area with standard cuts [9], and the dashed green line is the sum trigger implementation [10]. The two dotted black curves are the effective area functions used in this work, denoted CTA realistic (lower) and CTA optimistic (upper).

emission assumptions used in GPP10. In general, this model requires a significant departure from the extrapolated Band function, and implies the appearance of a separate high-energy spectral component.

\subsection{Telescope Properties}

As many of the properties of the CTA are indeterminate at the time of writing, we have relied on the design concept for the array described in [14], as well as reasonable extrapolations from the current generation of IACTs, particularly the MAGIC telescope. Our assumptions for realistic and optimistic effective area functions of CTA, shown in Figure 2, are based on configuration E, which assumes a central cluster of four 24-meter class telescopes that determine the low-energy sensitivity of the instrument. Sensitivity at energies above a few hundred $\mathrm{GeV}$, which is provided by more dispersed arrays of 12- and 7-meter class instruments, is not crucial to our results here, as most GRBs will occur at redshifts for which emission at these energies is strong attenuated by the EBL.

The transient and random nature of GRB emission represent the main difficulty in detecting emission from these sources. The satellite localization time of the event, transmission of the data to the ground, and slew time for the IACT all contribute to a total delay time for the commencement of observation. The localization time is dependent on the instrument and brightness of the GRB, but times of $<15 \mathrm{sec}$ are typical, and the transmission time is expected to be nearly instantaneous [15]. The largest class of telescopes in CTA, which provide coverage at the crucial low energies, are expected to have a slew time of 20 to 30 seconds. As a baseline assumption, we assume a total response time of 60 seconds in this work.

\section{Results and Discussion}

The GRB detection capabilities of the CTA can be described as the product of two independent factors: the detection efficiency, or probability that a randomly-selected GRB from our population occurring within the field-of-view of the telescope produces a detectable signal, and the rate at which the telescope is able to respond to triggers from satellite instruments within that field of 

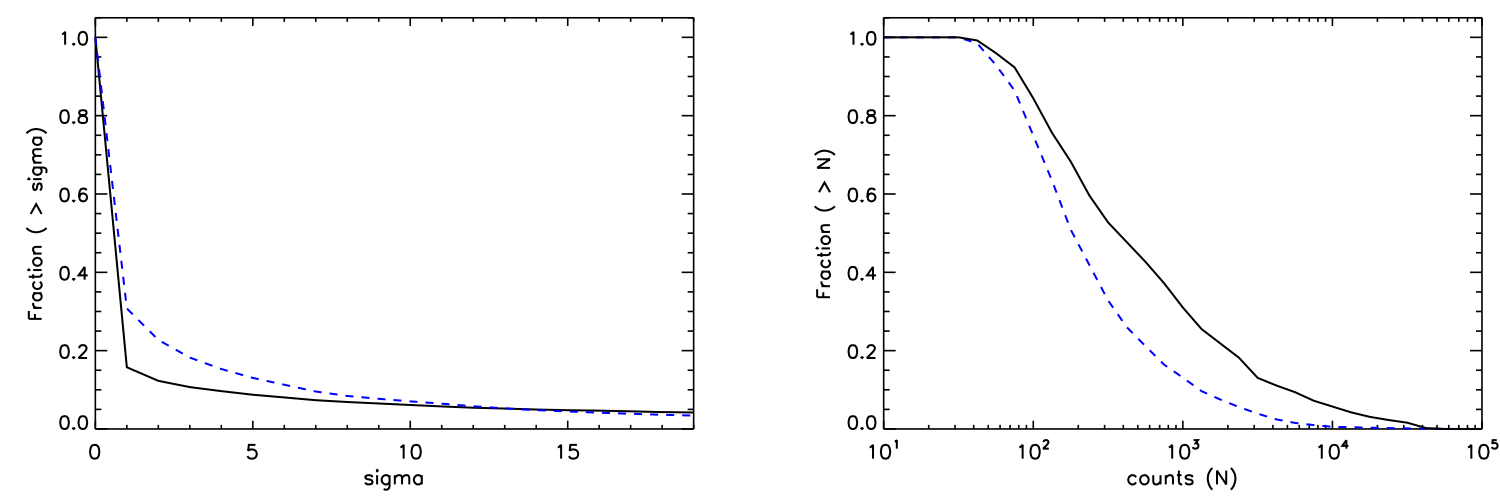

Figure 3: Left: The integral distribution of sigma values (significance of detection) for GRBs in our population. The solid line is for the direct extrapolation of Band functions ('bandex' model), from the distribution seen in BATSE GRBs, and the broken line is for the 'fixed model', using parameters described in the last section. Right: The integral distribution of counts above background, in the timescale bin with maximum sigma for each GRB that is detected using the criteria discussed in the text.

view. Our modeling of the former is done by simulating observations from the population of GRBs described in the previous section. For each GRB, we determine the integrated photon counts and expected background counts on timescales varying from 1 to $10^{4} \mathrm{sec}$. The significance of the detection is then calculated using the procedure described in [16]. The GRB is then assumed to be detected if the significance $(\sigma)$ is higher than 5 and at least 10 photons were seen above background in at least one timescale bin.

In Figure 3, we show results for an analysis done for a field of view $\theta_{\text {zenith }}<60$ deg, i.e., assuming the GRB occurs randomly on the sky within 60 deg of zenith. The curves in each plot are the integral probability distribution for the significance of the recorded gamma-ray signal, and the number of photons above background.

This baseline model predicts a detection efficiency of GRBs of $11 \%$ and $18 \%$ in the 'bandex' and 'fixed' models, respectively, for the realistic effective area function ( Figure 2) and $14 \%$ and $26 \%$ for the optimistic case. Corresponding numbers for the MAGIC standard cuts effective area are found to be $4.8 \%$ and $6.8 \%$. While these two models do predict somewhat different distributions in terms of significances for recorded signal and absolute photon counts, the predictions for detection efficiency using our above criterion $\left(\mathrm{N}_{\gamma} \geq 10 ; \sigma \geq 5\right)$ differ by less than a factor of 2 .

In the first panel of Figure 4, we show the overall impact of parameter $T_{\text {delay }}$ on detection efficiency, for the two effective area curves shown in Figure 2. The impact of VHE observation delay time due to GRB localization and telescope slew time is dependent on the assumed model for the GRB lightcurve at these energies, which we have modeled here as a $t^{-1.5}$ decay. In the right-hand side of Figure 4, we show how a higher value of the telescope energy threshold than the $20 \mathrm{GeV}$ assumed above would influence the detection efficiency. Effective area functions are assumed here to have the same shape as presented in Figure 2, but with a shift to higher energy by a constant factor, and the delay time set to $60 \mathrm{~s}$. As discussed in the introduction, GRB observations are strongly affected by spectral cutoffs due to extragalactic background light, and raising the telescope threshold energy reduces the redshift range over which GRBs are detectable. Detection 

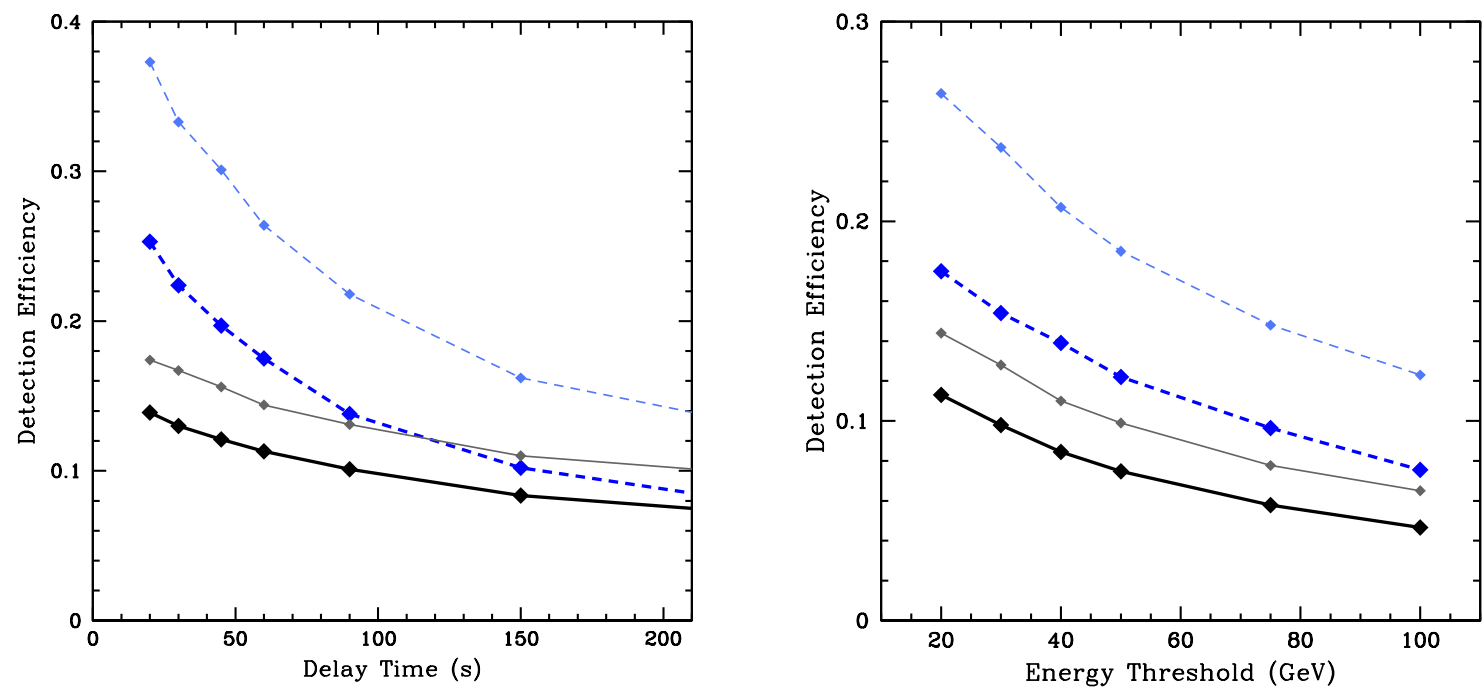

Figure 4: The effect on detection efficiency of varying instrument parameters. Left: The detection rate vs. observation delay time $\left(T_{\text {delay }}\right)$. The thick solid black and dashed blue lines are predictions from our bandex and fixed model, respectively, for the 'realistic' effective area function. Thinner grey and cyan lines show the corresponding results using the 'optimistic' effective area. Right: The effect on the overall detection efficiency of raising the energy threshold of each of our assumed effective area functions (Figure 2) from their initial values of $20 \mathrm{GeV}$.

efficiency is seen here vary strongly with energy threshold, for both spectral extrapolation models.

An approximate prediction for the trigger rate can be easily calculated using the alert rate from satellite instruments. The Swift satellite has historically observed GRBs at a rate of about 95/yr [17]. With a $10 \%$ duty cycle and a correction for the anti-solar bias present in Swift alerts (see discussion in [4]), a rate of $\sim 2$ GRBs per year within 60 degrees of zenith can be estimated. Our results for the realistic CTA effective area function suggest that a diligent GRB observation campaign could detect GRBs at a rate of one every 2-3 years $\left(0.36\right.$ and $0.60 \mathrm{yr}^{-1}$ for the bandex and fixed models), with great scientific impact. We note that the presence of a GeV-scale spectral cutoff, which we have not considered here, could reduce this rate significantly. Another upcoming experiment which could provide well-localized alerts in real time is the SVOM satellite [18], which is expected to detect GRBs at nearly the same rate as Swift, though the detected GRBs could be biased towards a softer, high redshift population, and may therefore be more difficult to detect at high energies.

In this proceeding we have described a method for determining the detection probability of GRBs with ground-based IACT instruments. An upcoming publication will include a detailed presentation of our model and results for the CTA experiment, as well as a careful test of our model against the GRB rate seen by Fermi LAT and upper limits set by current generation IACTs. Alerts from other satellite instruments, including Fermi GBM and the upcoming SVOM experiment, could contribute to the trigger rate and will be considered as well. 


\section{Acknowledgments}

RCG acknowledges a research fellowship from the SISSA Astrophysics Sector. RCG and JRP acknowledge Fermi Gamma Ray Telescope Theory grants.

\section{References}

[1] F. Aharonian et al., HESS observations of $\gamma$-ray bursts in 2003-2007, A\&A 495, 505-512 (2009)

[2] J. Albert, et al., MAGIC Upper Limits on the Very High Energy Emission from Gamma-Ray Bursts, ApJ 667, 358-366 (2007)

[3] D. Horan, Observations of Gamma-ray Bursts with VERITAS and Whipple, in International Cosmic Ray Conference 3, 1107-1110 (2008)

[4] R. C. Gilmore, F. Prada, and J. Primack, Modelling gamma-ray burst observations by Fermi and MAGIC including attenuation due to diffuse background light, MNRAS 402, 565-574 (2010)

[5] G. Ghisellini, G. Ghirlanda, L. Nava, and A. Celotti, GeV emission from gamma-ray bursts: a radiative fireball?, MNRAS 403, 926-937 (2010)

[6] D. Band et al., BATSE observations of gamma-ray burst spectra. I - Spectral diversity, ApJ 413, 281-292 (1993)

[7] R. C. Gilmore, P. Madau, J. R. Primack, R. S. Somerville, and F. Haardt, GeV gamma-ray attenuation and the high-redshift UV background, MNRAS 399, 1694-1708 (2009)

[8] J. Greiner et al., The redshift and afterglow of the extremely energetic gamma-ray burst GRB 080916C, A\&A 498, 89-94 (2009)

[9] J. Albert et al., VHE $\gamma$-Ray Observation of the Crab Nebula and its Pulsar with the MAGIC Telescope, ApJ 674, 1037-1055 (2008)

[10] M. Rissi et al., A New Sum Trigger to Provide a Lower Energy Threshold for the MAGIC Telescope, in IEEE Transactions on Nuclear Science 56, No. 6, 3840 (2009)

[11] C. D. Dermer, First Light on GRBs with Fermi, ArXiv:1008.0854.

[12] B. L. Dingus, EGRET Observations of $>30 \mathrm{MeV}$ Emission from the Brightest Bursts Detected by BATSE, Ap\&SS 231, 187-190 (1995)

[13] T. Le, and C. D. Dermer, Gamma-ray Burst Predictions for the Fermi Gamma Ray Space Telescope, ApJ 700, 1026-1033 (2009), ArXiv:0807.0355.

[14] The CTA Consortium, Design Concepts for the Cherenkov Telescope Array, ArXiv: 1008.3703

[15] D. Bastieri et al., The MAGIC Telescope and the observation of Gamma Ray Bursts, Nuovo Cimento C Geophysics Space Physics C 28, 711 (2005),

[16] T. Li, and Y. Ma, Analysis methods for results in gamma-ray astronomy, ApJ 272, 317-324 (1983)

[17] N. Gehrels, E. Ramirez-Ruiz, and D. B. Fox, Gamma-Ray Bursts in the Swift Era, ARA\&A 47, 567-617 (2009)

[18] D. Götz et al., SVOM: a new mission for Gamma-Ray Burst Studies, in American Institute of Physics Conference Series 1133, 25-30 (2009) 